\title{
EXTRAÇÃO SEQUENCIAL E ESPECIAÇÃO DE METAIS PESADOS NO DECORRER DO PROCESSO DE COMPOSTAGEM DE RESÍDUOS SÓLIDOS DOMICILIARES
}

\section{SEQUENTIAL EXTRACTION AND SPECIATION OF HEAVY METALS IN THE PROCESS OF COMPOSTING OF WASTE GARBAGE}

\section{José Carlos Chitolina ${ }^{1}$, Fábio Cesar da Silva ${ }^{2}$, Valter Barbieri ${ }^{3}$, Stefan Barradas Podsclan ${ }^{4}$}

${ }^{1}$ Diretor e Professor da Escola de Engenharia de Piracicaba - Fundação Municipal de Ensino de Piracicaba/FUMEP, Av. Monsenhor Martinho Salgot, 560 - Vila Areião,

13414-040 - Piracicaba, SP, Brasil. E-mail: jcchito@yahoo.com.br

${ }^{2}$ Pesquisador da Embrapa Informática Agropecuária, Professor do FATEP /IAT, Pósdoutorado no Departamento de Produção Vegetal da ESALQ/USP e da Universidade Politécnica de Madri, C. Postal 6041, Cidade Universitária Zeferino Vaz, 13.083-970

Campinas SP, Brasil, e-mail: fcesar@cnptia.embrapa.br

${ }^{3}$ Professor Doutor - Departamento de Engenharia de Biossistemas Área de Física e Meteorologia Universidade de São Paulo, Escola Superior de Agricultura Luiz de

Queiroz. Av. Pádua Dias, 11 13418-900 - Piracicaba, SP - Brasil. e-mail: vbarbier@esalq.usp.br

${ }^{4}$ Graduando em engenharia Agrícolas / Bolsista da Embrapa/CNPTIA - FeagriUnicamp Av. Candido Rondon, 501 Barão Geraldo - Cidade Universitária Zeferino Vaz CEP 13083-875 - Campinas, SP - Brasil. e-mail: stefan.podsclan@feagri.unicamp.br

\section{RESUMO}

A poluição ambiental por metais pesados, pela intensificação das atividades industriais, agrícolas e urbanização, em especial a geração de resíduos sólidos e a necessidade de tratá-los biologicamente consiste num problema crescente, responsável por sérios impactos ecológicos. Efetuou-se extração sequencial de metais pesados para avaliar a especiação de $\mathrm{Cu}, \mathrm{Cd}, \mathrm{Mn}, \mathrm{Ni}, \mathrm{Pb}$ e $\mathrm{Zn}$ no lixo orgânico durante a compostagem, permitindo o entendimento das transformações no processo e potenciais riscos ambientais destes elementos.Os extratores utilizados na extração sequencial modificam as propriedades que influenciam fortemente na interação do metal com a fase sólida, e as respectivas frações solúveis no início e final da compostagem, quanticadas por Espectrometria de Emissão ótica com fonte de plasma acoplado indutivamente - ICP OES. A sequencia dos extratores utilizada e as respectivas frações (início e fim da compostagem) foram: $\mathrm{MgCl}_{2}$ (fração solúvel - 5 e $2 \%$ para $\mathrm{Cu}$, 6 e $7 \%$ de $\mathrm{Mn} ;<1$ de $\mathrm{Zn}, 2$ e $3 \%$ de $\mathrm{Ni}, 0,7$ e $0,1 \%$ para $\mathrm{Pb} ; 24$ e $33 \%$ para $\mathrm{Cd}$ ); $\mathrm{CH}_{3} \mathrm{COONa}$ 
(carbonato - 4, 3\% para $\mathrm{Cu}, 24$ e $28 \%$ de $\mathrm{Mn}$; 25 e $20 \%$ para $\mathrm{Zn}, 6$ e $7 \%$ de $\mathrm{Ni}, 18$ e $13 \%$ a $\mathrm{Pb}, 37$, $33 \%$ para Cd); Hidroxilamina + HOA (reduzida / oxidada) - 4\% para $\mathrm{Cu}, 6,21 \%$ para $\mathrm{Mn}$ e 22 , $17 \%$ para $\mathrm{Zn}, 10,14 \%$ de Ni, 38, 21\% para $0 \%$ para $\mathrm{Pb}$ e $\mathrm{Cd}$ ) $; \mathrm{H}_{2} \mathrm{O}_{2}+\mathrm{NH}_{4} \mathrm{OAc}$ (orgânicos - 22, $41 \%$ para $\mathrm{Cu}, 3,4 \%$ de $\mathrm{Mn} ; 7,4 \%$ para $\mathrm{Zn}, 6,14 \%$ de $\mathrm{Ni}, 39,49$ e $0 \%$ para $\mathrm{Pb} \%$ para Cd) e $\mathrm{HNO}_{3}$ $+\mathrm{HClO}_{4}$ (porção restante - 65, 50\% para $\mathrm{Cu}, 61,40 \%$ de $\mathrm{Mn} ; 45,58 \%$ de $\mathrm{Zn} ; 76,62 \%$ de Ni, 4, $17 \%$ para $\mathrm{Pb} ; 38,34 \%$ para o $\mathrm{Cd}$ ). Os resultados mostraram que o processo de compostagem propicia que as maiores frações de $\mathrm{Cu}$ sejam orgânicas ou residuais, ou carbonato de $\mathrm{Mn}$ em formas reduzidas e as $\mathrm{Zn}$ permanecem na forma residual ou reduzida, Ni e Pb predominaram orgânicos ou residual e o $\mathrm{Cd}$ em residual ou na forma de carbonato, potencializando os riscos ambientais.

Palavras chave: Resíduos orgânicos. Compostagem. Risco ambiental.

\begin{abstract}
Environmental pollution by heavy metals caused by the intensification of industrial activities, agriculture and urbanization, especially the generation of solid waste and the necessity of treat them biologically is a growing problem, causing serious ecological impacts. It carried out sequential extraction for heavy metals speciation in organic trash during composting, objecting the understanding of chemical $\mathrm{Cu}, \mathrm{Mn}$ and $\mathrm{Zn}$ transformations and environmental risks. The extractors utilized in sequence and the respective fractions (beginning and ending of composting) were $\mathrm{MgCl}_{2}$ (soluble fraction $-5,2 \%$ to $\mathrm{Cu} ; 6,7 \%$ to $\mathrm{Mn}$; $<1$ to $\mathrm{Zn} ; 2,3 \%$ to $\mathrm{Ni} ; 0.7,0.1 \%$ to $\mathrm{Pb} ; 24,33 \%$ to $\mathrm{Cd}$ ); $\mathrm{CH}_{3} \mathrm{COONa}$ (carbonate $-4,3 \%$ to $\mathrm{Cu} ; 24,28 \%$ to $\mathrm{Mn} ; 25,20 \%$ to $\mathrm{Zn} ; 6,7 \%$ to $\mathrm{Ni} ; 18,13 \%$ to $\mathrm{Pb} ; 37,33 \%$ to $\mathrm{Cd}$ ); Hidroxilamina+HOA (reduced/ oxidated) $-4 \%$ to $\mathrm{Cu}, 6,21 \%$ to $\mathrm{Mn}$ and 22 , $17 \%$ to $\mathrm{Zn} ; 10,14 \%$ to $\mathrm{Ni}, 38,21 \%$ to $\mathrm{Pb}$ and $0 \%$ to $\mathrm{Cd}$ ); $\mathrm{H} 2 \mathrm{O} 2+\mathrm{NH} 4 \mathrm{OAc}$ (organic $-22,41 \%$ to $\mathrm{Cu}, 3,4 \%$ to $\mathrm{Mn} ; 7,4 \%$ to $\mathrm{Zn} ; 6,14 \%$ to $\mathrm{Ni}, 39,49 \%$ to $\mathrm{Pb}$ and $0 \%$ to $\mathrm{Cd}$ ) and $\mathrm{HNO}_{3}+\mathrm{HClO}_{4}$ (remaining portion $-65,50 \%$ to $\mathrm{Cu}, 61,40 \%$ to $\mathrm{Mn} ; 45,58 \%$ to $\mathrm{Zn} ; 76,62 \%$ to $\mathrm{Ni}, 4,17 \%$ to $\mathrm{Pb}$; $38,34 \%$ to $\mathrm{Cd}$ ). The results show that composting process provides that the biggest fractions of $\mathrm{Cu}$ is organic or residual, $\mathrm{Mn}$ in carbonate or reduced forms and the $\mathrm{Zn}$ remains in the residual or reduced forms, $\mathrm{Ni}$ and $\mathrm{Pb}$ are organic or residual and the $\mathrm{Cd}$ remains in the residual or carbonate forms, offering bigger ambient risks.
\end{abstract}

Keywords: Organic wastes. Composting. Ambient risk.

\title{
1. INTRODUÇÃO
}

Dentre os processos de reciclagem do lixo urbano orgânico, a compostagem, é uma das melhores formas para minimizar o acúmulo desse material em lixões ou aterros, que, além de desocupar uma área que poderia ser utilizada para fins mais nobres, não oferece riscos à saúde humana (SILVA et al., 2009). Na Europa, o uso de composto de resíduos orgânicos para melhorar a fertilidade do solo data do Império Romano e era muito popular entre agricultores da Idade Média (PARR e HORNICK, 1992; BLUM, 1992; SILVA et al., 2009). A concentração da população nos centros urbanos, como resultado da Revolução Industrial, no século XVII, representou uma ruptura no processo de retorno de nutrientes ao solo, na forma de restos alimentares, fezes e vestuário. Quando este é tratado por meio da compostagem o produto derivado do processo é chamado composto de lixo urbano ou de resíduo sólido domiciliar (CLU), que após a $2^{\text {a }}$ Guerra Mundial teve sua utilização na agricultura reduzida gradualmente, devido a crescente produção de fertilizantes químicos somado à facilidade de transporte e manuseio desses fertilizantes pelos agricultores (SILVA et al., 2009). Para as substâncias orgânicas de interesse ambiental (metais pesados) é fundamental conhecer-se apriori os teores totais naturais dos metais pesados nos compartimentos ambientais, e os valores tornam-se de referência de qualidade (CETESB, 2001). 
Nas cidades modernas existem diversas fontes antropogênicas de metais pesados no ambiente são fertilizantes, resíduos sólidos, águas contaminadas, entre outras. já não há mais lugar para dispor os resíduos sólidos gerados, que sugere os gestores públicos visualizarem que a compostagem é uma alternativa viável para reciclagem da fração orgânica desse material na agricultura (MALAVOLTA, 1994; SILVA et al., 2009), pois de um lado melhora alguns atributos químico, físico e biológico do solo, e de outro, contribui para aliviar a carga poluidora e aumentar a vida útil dos aterros sanitários (DEELSTRA, 1989).

Em um processo de compostagem é necessário que seja precedida pela coleta seletiva, com o objetivo de evitar-se ao máximo a presença de contaminantes (material não-orgânico) no composto (EMERSON, 2004). Dentre os poluentes, destaca-se a presença de metais pesados (SILVA et al., 2005) que podem estar no ambiente, os quais são potencialmente tóxicos às plantas e aos animais superiores são $\mathrm{As}, \mathrm{Cd}, \mathrm{Pb}, \mathrm{Hg}, \mathrm{Co}, \mathrm{Cu}, \mathrm{Cr}$, Ni, Se e $\mathrm{Zn}$ (NELLESSEN e FLETCHER, 1993), sendo o Cd, Ni e Zn considerados mais preocupantes em fitotoxidez na aplicação de resíduos em solos (MCBRIDE, 1994; CAMARGO et al., 2001).

O conhecimento da interação entre o processo de compostagem com os metais pesados é fundamental, como uma opção de tratamento de mitigação, porém, não basta conhecer as quantidades totais, mas é necessário saber sua especiação iônica (LÓPES-MOSQUERA et al., 2000) e a sua reatividade no ambiente (McBride, 1994), as quais se alteram no decorrer do processo (SILVA et al., 2009).

A especiação de metais pesados tem sido estudada na literatura para compreender a dinâmica destes metais nos compartimentos ambientais e nas transformações biológicas (TESSIER et al., 1979; ALLOWAY, 1990), sendo a técnica de extração sequencial bastante adequada para estudos ambientais e as distintas frações, possibilita a avaliação do potencial tóxico de cada elemento (SPOSITO et al., 1982; CHANG et al., 1984).

\section{MATERIAL E METODOS}

No método da extração sequêncial, uma mesma amostra é submetido a extrações continuas, em frações definidas, cujo poder de extração aumenta a cada fração. Foi realizada a extração sequencial do resíduo solido orgânico domiciliar durante o processo de compostagem, no inicio aos 30 dias ("pré-composto" ou crú) e maturado ou estabilizado aos 180 dias, os quais foram provenientes de duas unidades paulistas, as quais utilizam-se do processo DANO (SILVA et al., 2005), localizadas em São Mateus (capital) e Santo André. O método de Tessier et al. (1979) foi utilizado para a extração sequencial, visando o entendimento das transformações químicas do $\mathrm{Cu}$, $\mathrm{Cd}, \mathrm{Mn}, \mathrm{Ni}, \mathrm{Pb}$ e $\mathrm{Zn}$ no decorrer da compostagem. A identificação das diferentes formas e fases na qual um elemento ocorre, defini-se como especiação do metal que relaciona a biodisponibilidade. Os extratores utilizados na extração seqüêncial modificam as propriedade que influenciam fortemente na interação do metal com a fase sólida, e as respectivas frações solúveis no início e final da compostagem que foram: $\mathrm{MgCl}_{2}$ (fração solúvel/trocável); $\mathrm{CH}_{3} \mathrm{COONa}$ (carbonatos); Hidroxilamina $+\mathrm{HOA}$ (redutível/oxidável); $\mathrm{H}_{2} \mathrm{O}_{2}+\mathrm{NH}_{4} \mathrm{OAc}$ (orgânico) e $\mathrm{HNO}_{3}+\mathrm{HClO}_{4}$ (residual) e quanticadas por método analitico por Espectrometria de Emissão ótica com fonte de plasma acoplado indutivamente, ICP OES (SILVA, 2009).

A percentagem de recuperação dos metais pesados é a relação da somatória de todas as frações obtidas no fracionado seqüencial dos CLU, em relação ao teor total obtida em digestão nítrico-perclórica (SILVA, 2009). Nos extratos determinaram-se os metais por ICP OES. Desta forma, obtêm-se as informações a respeito das frações química (quantidade de metal que é extraída por um reagente especifico), sendo as fases (espécies química do metal que um reagente deve extrair) e possibilitando avaliar o potencial tóxico de cada elemento (SPOSITO et al., 1982). 


\section{RESULTADOS E DISCUSSÃO}

O primeiro aspecto observado nos resultados das amostras de metais pesados dos compostos de lixo das três usinas foi o teor total altos de $\mathrm{Cd}$, na maioria das amostras de Santo André $\left(\mathrm{L}_{2}\right)$ e em parte de São Mateus ( $\left.\mathrm{L}_{1}\right)$, isto é, acima de $5 \mathrm{mg} \cdot \mathrm{kg}^{-1}$ (SILVA et al., 2009).

O segundo aspecto, considerando-se as duas usinas, os valores de recuperação das frações desses metais ainda são limitados, especialmente em função da carga orgânica originária da unidade no inicio da compostagem, cujas variações nas porcentagens da somatória das frações em relação ao total que foram as seguintes: $\mathrm{Cu}$ de 31,3 a 93\%; Mn de 27 a 79\%; Fe de 43 a 78\% e Zn de 26 a 56\% (Tabela 1).

Nas Figuras 1 e 2, verifica-se que de 20 a 32\% do Cd total é prontamente solúvel, acrescido ao fato de mais 20 a 30\%, que aumenta a possibilidade de reagirem no ambiente também. Tal fato é evidenciado pelo $\mathrm{Ni}$, onde a fração orgânica é função do resíduo orgânica domiciliar que predomina na URC $\left(\mathrm{L}_{1}\right.$ ou $\left.\mathrm{L}_{2}\right)$.

TABELA 1 - Percentagem de recuperação e do total dos metais pesados nas frações pelo método de fracionado seqüencial dos compostos orgânicos produzidos nas URCs Paulistanas (Usinas S. Matheus ${ }^{1}\left(\mathrm{~L}_{1}\right)$ e S. André ${ }^{2}-\mathrm{L}_{2}$ )

\begin{tabular}{cccc}
\hline Metal pesado & Teor Total & \multicolumn{2}{c}{ RECUPERAÇÃO( $\left.{ }^{3}\right), \%$} \\
\cline { 2 - 3 } & mg.kg ${ }^{-1}$ & $(\mathrm{~L} 1)$ & $(\mathrm{L} 2)$ \\
\hline $\mathrm{Cu}$ & $304-318$ & $40-44$ & $41-56$ \\
$\mathrm{Cd}$ & $3-10$ & $79-82$ & $55-65$ \\
$\mathrm{Mn}$ & $301-430$ & $47-73$ & $28-68$ \\
$\mathrm{Ni}$ & $79-114$ & $44-52$ & $23-26$ \\
$\mathrm{~Pb}$ & $164-200$ & $98-102$ & $44-78$ \\
$\mathrm{Zn}$ & $510-1249$ & $56-43$ & $42-40$ \\
\hline
\end{tabular}

(3) Rec. $\%$ = soma das frações/ teor total $\mathrm{x} 100$

Nas Figuras 1 e 2, nota-se que os extratores utilizados na seqüência e as respectivas frações no início e final da compostagem, respectivamente, foram: $\mathrm{MgCl}_{2}$ (fração solúvel/trocável 5 e $2 \%$ para $\mathrm{Cu} ; 6$ e $7 \%$ para $\mathrm{Mn} ; 2$ e 3\% para $\mathrm{Ni} ; 0,7$ e $0,1 \%$ para $\mathrm{Pb}$ e 24 e $33 \%$ para $\mathrm{Cd}$ e $>1$ para $\mathrm{Zn}$ ); $\mathrm{CH}_{3} \mathrm{COONa}$ (carbonatos 4 e $3 \%$ para $\mathrm{Cu}, 24$ e $28 \%$ para $\mathrm{Mn} ; 6$ e $7 \%$ para $\mathrm{Ni}, 18$ e $13 \%$ para $\mathrm{Pb}$ e 37 e 33\% para Cd e 25 e 20\% para Zn); Hidroxilamina+HOA (redutível/oxidável $4 \%$ para Cu, 6 e $21 \%$ para $\mathrm{Mn} ; 2210$ e $14 \%$ para $\mathrm{Ni}, 38$ e $21 \%$ para $\mathrm{Pb}$ e $0 \%$ para $\mathrm{Cd}$ e $17 \%$ para $\mathrm{Zn}$ ); $\mathrm{H} 2 \mathrm{O} 2+\mathrm{NH} 4 \mathrm{OAc}$ (orgânico 22 e $41 \%$ para $\mathrm{Cu}, 3$ e 4\% para Mn; 6 e 14\% para Ni, 39 e $49 \%$ para $\mathrm{Pb}$; $0 \%$ para $\mathrm{Cd}$ e 7 e $4 \%$ para $\mathrm{Zn}$ ) e $\mathrm{HNO}_{3}+\mathrm{HClO}_{4}$ (residual 65 e $50 \%$ para $\mathrm{Cu}, 61$ e $40 \%$ para $\mathrm{Mn} ; 76$ e $62 \%$ para $\mathrm{Ni}, 4$ e $17 \%$ para $\mathrm{Pb}$ e 38 e $34 \%$ para $\mathrm{Cd}$ e 45 e $58 \%$ para $\mathrm{Zn}$ )

Os resultados mostram que a técnica de fracionamento permitiu avaliar o processo de compostagem. Utilizando-se da regra de três, onde a somatória das frações ( $\sum$ Frações) de cada metal é considerado 100\% (Tabela 1). Deve ser ressaltado, a maioria dos métodos de extração sequencial foram desenvolvido para estudos em sedimentos (SHANNON e WHITE, 1991), como a 
proposta por Tessier et al. (1979), o que requer uma gradual evolução metodologica. Todavia, apesar de algunas limitações observadas no estudo, a extração sequencial é útil na avaliação de formas quimícas dos metais no ambiente, fornecendo dados relevantes quanto à mobilidade e à biodisponibilidade de nutrientes e de poluentes metálicos (JORDÃO e NICKLESS, 1989).

Quanto à distribuição de metais frações da extração seqüencial do composto pode-se, de modo geral, dizer que: o Cd encontra-se principalmente nas frações trocável e carbonato de 38 e $34 \%$ do total e também residual; o Ni encontra-se na fração residual de 76 e $62 \%$ do total, em sua maior parte, havendo uma tendência na compostagem a formar uma fração orgânica (Figura 2); o $\mathrm{Pb}$ encontra-se predominando nas frações carbonatos e redutível com 39 e $49 \%$ e em parte no decorrer do processo tende a residual (SPOSITO et al., 1982). As percentagens das frações não se alteram entre as usinas (L1 e L2), mesmo para os valores de concentração totais diferentes (Figuras 1 e 2).

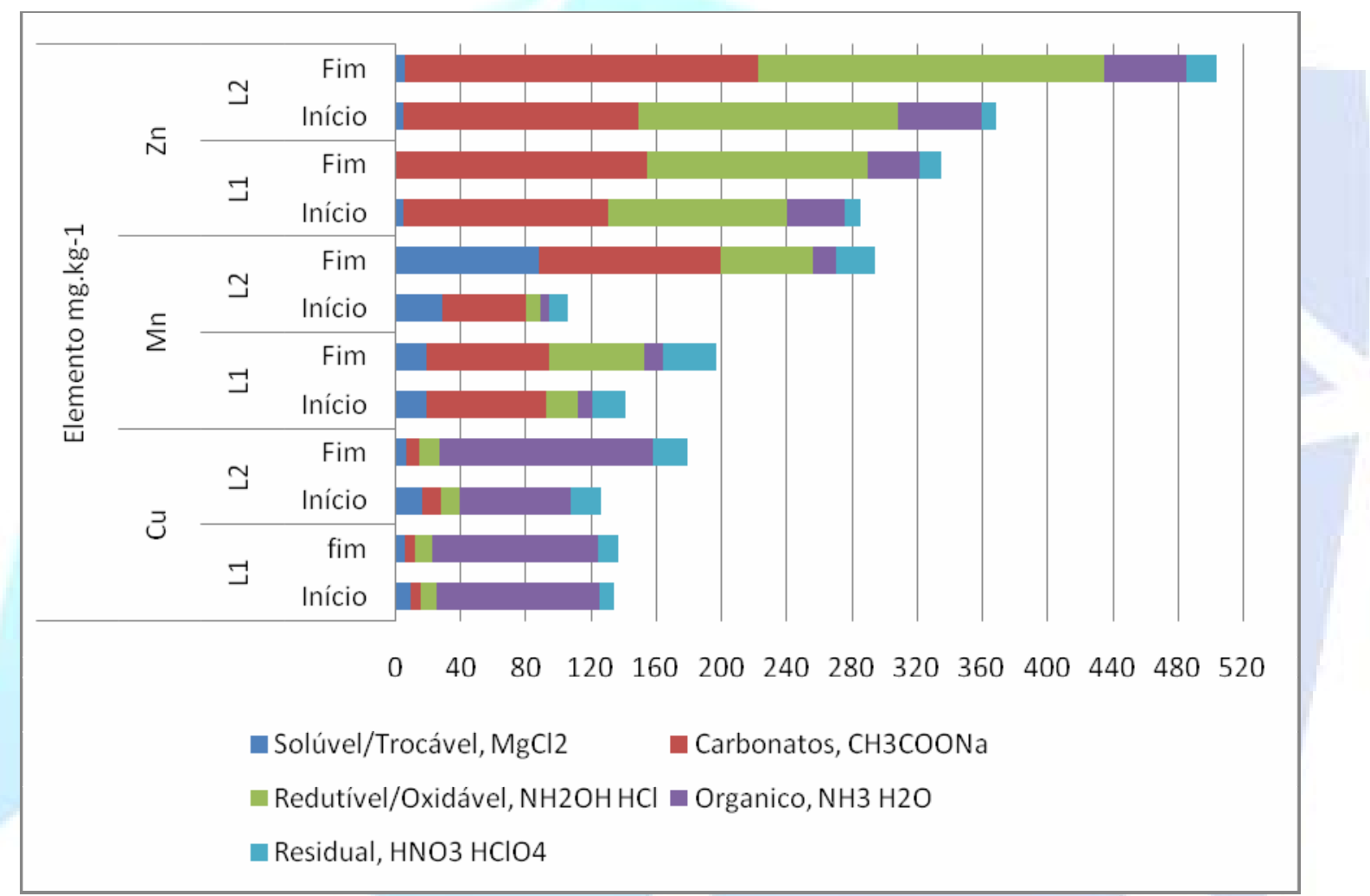

FIGURA 1. Dinâmica Quantitativa de $\mathrm{Cu}, \mathrm{Mn}$ e $\mathrm{Zn}$ na compostagem do lixo domiciliar, em mg.kg ${ }^{-1}$, no início ("crú") aos 30 dias e após aos 180 dias ("maturado"), nas unidades (Usinas S. Matheus - L1 e Santo André - L2).

$\mathrm{Na}$ Figura 1, nota-se que o $\mathrm{Cu}$ esteve ligado à forma orgânica em 70 a $75 \%$ e de 15 a 20\% está residual desse total, devido a sua afinidade de complexação com compostos orgânicos; (ELLIOT et al., 1986). O Mn esta mais ligado as frações carbonatos em 39\% e a óxidos de Fe e Al. Já o Zn do composto encontrou-se na sua maior parte ligado aos carbonatos de 45 a $64 \%$ e como redutível ou oxidável, na ordem de $50 \%$ do total, tendendo a fração carbonato aumentar no decorrer do processo, indicam a potencial disponibilidade do elemento para as plantas. Comparando-se as frações dos metais no inicio e no final da compostagem, não foi possível verificar uma migração importante desses micronutrientes do CLU de uma forma extraída pelos vários métodos, em função do tempo de compostagem, exceto o $\mathrm{Zn}$ que foi para formas mais fitodisponível (CHANG et al., 1984). 


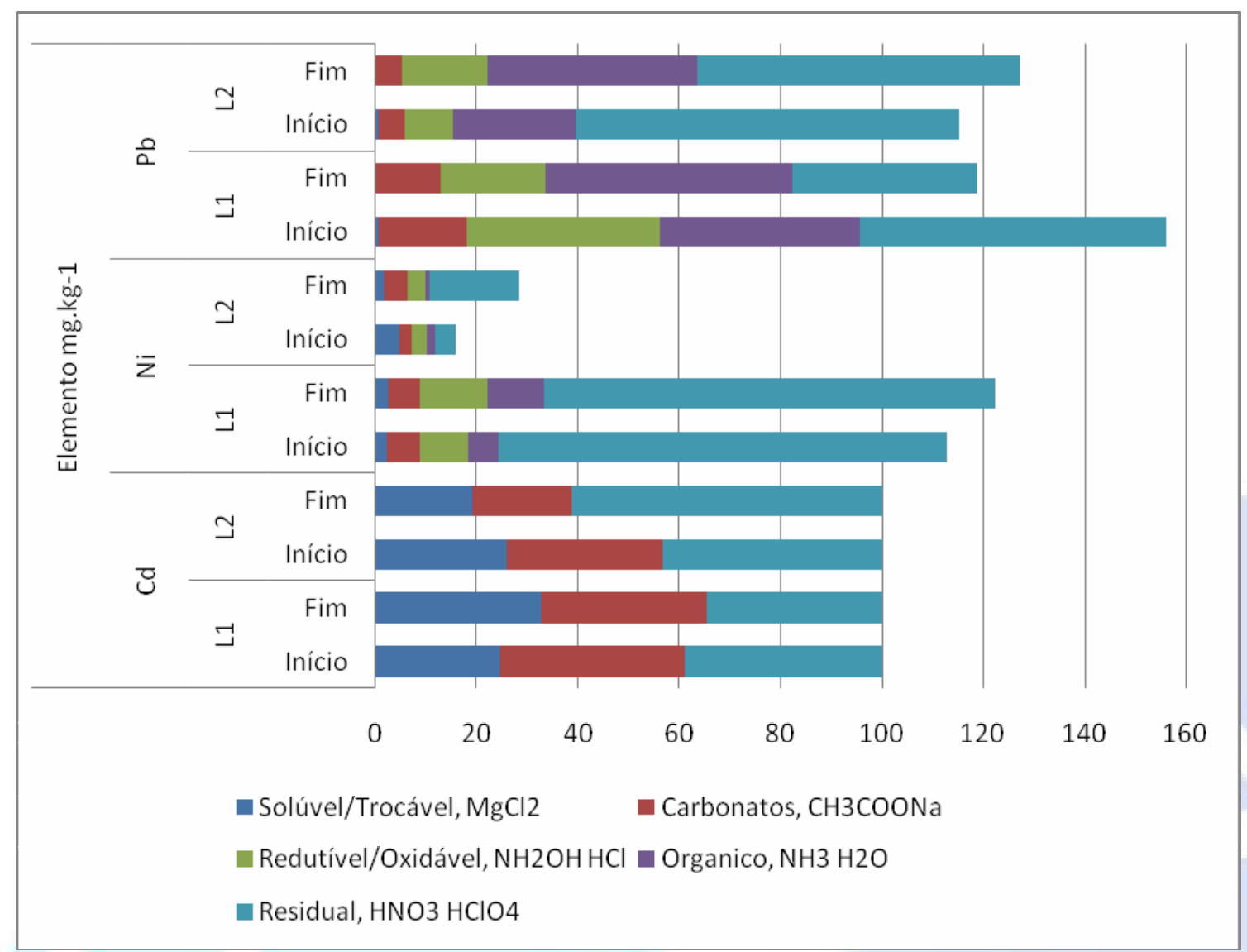

FIGURA 2. Dinâmica Quantitativa de Cd, Ni e na compostagem do lixo domiciliar, em mg. $\mathrm{kg}^{-1}$, no início ("crú") aos 30 dias e após aos 180 dias ("maturado"), nas unidades (Usinas S. Matheus - L1 e Santo André L2).

Talvez, os erros inerentes a técnica de fracionamento de metais tenha mascarado essa tendência para esses metais pesados do CLU, que atribui na literatura, refere-se às incertezas (SPOSITO et al., 1982), como:

a) temperatura e tempo de secagem da amostra;

b) tempo de contato de cada extrator com a amostra;

c) volume do frasco extrator;

d) tempo e método de agitação;

e) temperatura ambiente;

f) falta de seletividade de alguns extratores, e

g) redistribuição (readsorção) dos metais entre as fases na extração.

\section{CONCLUSÕES}

A técnica de fracionamento seqüencial do lixo orgânico durante compostagem, permite entender as transformações do $\mathrm{Cu}, \mathrm{Cd}, \mathrm{Mn}, \mathrm{Ni}, \mathrm{Pb}$ e $\mathrm{Zn}$ durante o processo e associar ao seus riscos ambientais. Sendo, que o Cd e Mn permanecem nas formas carbonato ou residual, oferecendo maior risco ambiental. Todavia, a técnica merece ainda um aprimoramento e a sua eficiência de 
recuperação é muito influenciada pela complexidade da carga orgânica contida no composto da unidade de origem.

\section{REFERÊNCIAS}

BLUM, B. Composting and the Roots of the Sustainable Agriculture. Agricultural History, v. 66, n.2, p171-188, 1992.

CAMARGO, O.A.; ALLEONI, L.R.F.; CASAGRANDE, J.C. Reações dos micronutrientes e elementos tóxicos no solo. In: FERREIRA, M.E.; CRUZ, M.C.P.; RAIJ, B.van; ABREU, C.A. Micronutrientes el elementos tóxicos na agricultura. Jaboticabal: CNPq; Fatesp; Potafos, 2001. cap.5, p.89-117.

CHANG, A.C.; GRANATO, T.C.; PAGE, A.L. A methodology for establishing phytotoxicity criteria for chromium, cooper, nickel, and zinc in agricultural land aplication of municipal sewage sludges. Journal Environ. Quality, v.21, p.521-536, 1992.

CETESB - COMPANHIA DE TECNOLOGIA E SANEAMENTO AMBIENTAL. Relatório de estabelecimento de valores orientadores para solos e águas subterrâneas no Estado de São Paulo. São Paulo: Companhia de Tecnologia e Saneamento Ambiental, 2001. 247p. (Relatório Técnico CETESB).

DEELSTRA, T. Can cities survive: solid waste management in urban environments. AT Source, v.18, n. 2, p. 21-27, 1989.

EMERSON, D. Single Stream Vs. Source Separated Recycling. BioCycle, v. 45, n.3, p. 22, 2004.

JORDÃO, C.P.; NICKLESS, G. Chemical associations of $\mathrm{Zn}, \mathrm{Cd}, \mathrm{Pb}$ and $\mathrm{Cu}$ in soils and sediments determined by sequential extraction technique. Environmental Technology Letters, Bristol, v.10, p.743-752, 1989.

LÓPES-MOSQUERA, M. E.; MIORÓN, C.; CARRAL, E. Use of dairy-industry sludge as fertilizer for grasslands in northwest Spain: heavy metal level $\mathrm{n}$ the soil and plant. Resources Conservation and Recycling, Amsterdam, v. 30, p. 95-109, 2000.

MALAVOLTA, E. Fertilizantes e seu impacto ambiental: micronutrients e metais pesados, mitos, mistificações e fatos. São Paulo: ProduQuimica, 1994, 153p.

McBRIDE, M. B. Environmental chemistry of soils. New York: Oxford University Press, 1994. 406p.

NELLESSEN, J.E.; FLETCHER, J.S. Assesment of published literature on the uptake.

Accumulation, and translocation of heavy metals by vascular plants. Chemosphere, Oxford, v.27, p.1669-1680. 1993.

PARR, J. F., HORNICK, S.B. Agricultural use of organic amendments: A historical perspective. American Journal of Alternative Agriculture, v.7, n. 4, p.181-189, 1992. 
SHANNO, R. D.; WHITE, J. R. The selectivity sequential extraction procedure for the determination of iron hydroxides and iron sulfites in lake sediments. Biogeochemistry, Dordrecht, v. 14, p. 193-208, 1991.

SILVA, F. C. da; CHITOLINA, J. C.; BALLESTERO, S. D.; VOIGTEL, S.D.S.; MELO, J. R.B. de Processos de produção de composto de lixo e a sua qualidade como fertilizante organico. Holos Environmental, Rio Claro, v. 5, n. 2, p. 121-136, 2005.

SILVA, F.C. Manual de analises químicas de solos, plantas e fertilizantes. 2.(Ed.) Brasília. Embrapa, 2009. 627 p.

SILVA, F.C.; RODRIGUES, M.S.; BARREIRA, L.P. Gestão Pública de Resíduo Sólido Urbano: Compostagem e Interface Agro-Florestal. 1. (ed.) Botucatu. Fepaf, 2009. 204 p.

SPOSITO, G.; LUND, L. J.; CHANG, A. C. trace metal chemistry in arid-zone field soils amended with sewage sludge. I. Fraction of $\mathrm{Ni}, \mathrm{Cu}, \mathrm{Zn}, \mathrm{Cd}$ and $\mathrm{Pb}$ in solid phases. Soil Science Society of America Journal, Madison, v. 46, p. 260-264, 1982.

TESSIER, A.; CAMPBELL, P.G.G.; BISSON,M. Sequential extraction procedure for the speciation of perticulate trace metals. Analytical Chemistry, v.51, n.7, p.844-851, 1979. 